\title{
TREATMENT OF WASTEWATER CONTAINING RHODAMINE BY ADVANCED OXIDATION PROCESS (AOP): FENTON, PHOTO-FENTON AND OZONATION
}

\author{
São José, cep 99052-900, Passo Fundo - RS, Brasil \\ (fone: +55 5433168100 ) \\ * Calina Ninfa Grando \\ e-mail: calinagrando@gmail.com
}

GRANDO, Calina Ninfa*; DIEHL, Matheus José Gomes²; MISTURA, Clóvia Marozzin³;

1,2 Universidade de Passo Fundo, Faculdade de Química, Departamento de Pesquisa em Química, BR, 285

\section{RESUMO}

A necessidade de conservação dos recursos naturais, assim como possíveis riscos à saúde humana, têm motivado esforços para minimizar problemas de contaminação. As operações de tingimento e lavagem de ágatas demandam a utilização de consideráveis volumes de água e produzem efluentes líquidos com coloração, além da possibilidade de alta toxicidade. Os POAs têm sido extensivamente avaliados, principalmente devido à sua capacidade de degradar um grande número de substâncias resistentes através de procedimentos de custo relativamente baixos e de simples operação. Estes processos se baseiam na geração do radical hidroxila $\left(\mathrm{OH}^{*}\right)$, que possui amplo potencial de oxidação. Obteve-se excelentes resultados na degradação da cor com os três tratamentos.

Palavras-chave: degradação; corante; mineral.

\section{ABSTRACT}

The necessity for the conservation of natural resources and possible risks to human health have motivated efforts to minimize contamination problems. The agate dyeing and washing operations demand the utilization of considerable volumes of water and yield colored wastewater in addition to the possibility of high toxicity. The Advanced Oxidation Process (AOP) have been extensively evaluated, mainly due to its high capacity for degrading a large number of resistant substances with relatively low cost procedure and simple operation. These processes are based upon the formation of the hydroxyl radical $\left(\mathrm{OH}^{\circ}\right)$; that has a wide potential for oxidation. All three treatments yielded excellent results in color degradation.

Keywords: degradation; dye; mineral.

PERIÓDICO TCHÊ QUÍMICA • www.periodico.tchequimica.com • Vol. 13 N. 26.

• ISSN 1806-0374 (impresso) • ISSN 1806-9827 (CD-ROM) • ISSN 2179-0302 (meio eletrônico)

(C) 2016. Porto Alegre, RS. Brasil 
INTRODUÇÃO

O Brasil é reconhecido internacionalmente como um dos principais produtores, tanto pela variedade quanto pela quantidade de gemas encontradas em seu subsolo. Atualmente, estima-se que o país seja responsável pela produção de cerca de um terço do volume das gemas do mundo (IBGM, 2015). O setor de gemas e joias no Rio Grande do Sul (RS) é considerado um dos cinco aglomerados do setor do país, envolve desde atividades de extração mineral, nas jazidas existentes no estado, até a produção e comercialização do produto final (APL, 2015).

Em Soledade e Ametista do Sul, as matérias-primas mais utilizadas são a ágata e a ametista, que constituem os diversos produtos derivados, tais como acessórios, pulseiras, colares, relógios etc., (ZANATTA, 2014), e sendo este o veio principal da economia local desses municípios.

Para as pedras tingidas artificialmente, o processo apresenta uma etapa de tingimento onde as pedras recebem tratamento específico para a cor desejada (SENAI, 2008). As

operações de tingimento de ágatas e lavagem das pedras demandam a utilização de consideráveis volumes de águas e são responsáveis pela produção de efluentes líquidos com coloração e, normalmente, alta toxicidade (MORAES, MISTURA e OLIVEIRA, 2011).

Além dos aspectos indesejáveis da cor, muitos corantes usados são nocivos ao homem e ao ambiente podendo apresentar alta acidez, sais dissolvidos, íons de metais pesados, surfactantes e matéria orgânica na forma de moléculas corantes complexas (SALAMONI, 2008; PERALTA-ZAMORA, et al., 1997).

Ao diminuir a transparência da água $e$ impedir a penetração da radiação solar, estes rejeitos coloridos diminuem a atividade fotossintética e provocam distúrbios na solubilidade dos gases, causando danos nas guelras e brânquias dos organismos aquáticos, pondo em risco a estabilidade desses ecossistemas desses ecossistemas e a vida em seu torno. Nos processos de degradação parcial em ambientes naturais e nas etapas de biotransformação nos recursos hídricos, podem gerar substâncias com propriedades carcinogênicas (ZANONI e CARNEIRO, 2001).
Os corantes presentes nos efluentes de indústrias são caracterizados por possuírem anéis aromáticos e um ou mais grupos cromóforos como, por exemplo, os grupamentos anatraquinona, azo, nitroso e, os que são estudados neste projeto, os xantênicos. (MARMITT, PIROTTA e STÜLP, 2010).

$\mathrm{O}$ corante Rodamina $\mathrm{B}$ pertence à classe dos corantes xantenos, um corante catiônico vermelho básico, que é altamente solúvel em água, metanol e etanol. Inicialmente, foi amplamente utilizado como corante na indústria têxtil e indústrias de plástico, e também é um corante fluorescente conhecido e aplicado na indústria química analítica e estudos biológicos (Ql, et al, 2014). O Xanteno é a base de uma classe de corantes, como por exemplo a fluoresceína, as eosinas, e as rhodaminas que são derivadas desta estrutura. Tendem a ser fluorescentes, resultando em corantes brilhantes, de amarelos rosados a vermelhos azulados (PALIWAL, 2013).

Os processos oxidativos avançados (POAs) têm sido extensivamente avaliados, principalmente devido à sua capacidade de degradar um grande número de substâncias resistentes através de procedimentos de custo relativamente baixo e de simples operação. Estes processos se baseiam na geração in situ do radical hidroxila $\left(\mathrm{OH}^{*}\right)$, que possui amplo potencial de oxidação (VIANNA, TORRES e AZEVEDO, 2008).

Processos que potencializam $0 \mathrm{OH}^{\cdot}$ incluem as reações de $\mathrm{H}_{2} \mathrm{O}_{2}$ e UV com reagente de Fenton e absorção de UV por um óxido de metal semicondutor suspenso na água e agindo como um catalisador (METCALF e EDDY, 2003, p. 1200).

As reações de radicais são não seletivas e instantâneas (LANGLAIS et al., 1991 apud GOI, 2005) a velocidade de reação é constante para reações de radicais e são de várias ordens de grandeza mais elevada do que para o ozônio molecular, mas, devido à elevada reatividade, radicais que são formados e reações indesejáveis com compostos da matriz, são desperdiçados por exemplo, com carbonatos e íons de bicarbonato (HABERL et al., 1993, apud GOI, 2005).

Assim a responsabilidade ambiental parte de um processo gradativo de conscientização, que se positiva de forma individual, por meio do

PERIÓDICO TCHÊ QUÍMICA • www.periodico.tchequimica.com • Vol. 13 N. 26 - ISSN 1806-0374 (impresso) • ISSN 1806-9827 (CD-ROM) • ISSN 2179-0302 (meio eletrônico) 
qual são realizadas práticas visando à diminuição do impacto causado na natureza (SILVA, et al. 2014)

\section{MATERIAL E MÉTODOS}

O objetivo de qualquer processo oxidativo avançado como tratamento químico, é o de gerar, ampliar e utilizar o radical livre hidroxila $\left(\mathrm{OH}^{\circ}\right)$, um forte agente oxidante, capaz de destruir compostos e micropoluentes orgânicos que não podem ser oxidados por oxidação química convencional (CAVALCANTI, 2012).

Foi preparado efluente simulado com 20 $\mathrm{mg} \mathrm{L}^{-1}$ do corante Rodamina B para avaliar a degradação dos tratamentos por POAs: Fenton, foto-Fenton e ozonização. Comparou-se os métodos a fim de verificar qual dos tratamentos apresenta maior eficiência com $20 \mathrm{mg}$ do corante, medido em balança analítica e diluído em água de osmose reversa em balão volumétrico. Para os tratamentos Fenton e fotoFenton foi utilizado peróxido de hidrogênio $\left(\mathrm{H}_{2} \mathrm{O}_{2}\right)$, que por sua vez foi titulado com permanganato de potássio a fim de verificar sua real concentração.

\subsection{Titulação do peróxido de hidrogênio}

A concentração de peróxido de hidrogênio foi determinada por permanganometria. Para isso foi preparada uma solução de permanganato de potássio $0,0225 \mathrm{~mol} \mathrm{~L}^{-1} \mathrm{em}$ balão de $200 \mathrm{~mL}$. O oxalato de sódio seco em estufa por uma hora. Em três erlenmeyers foram medidos $0,15 \mathrm{~g}$ em balança analítica e adicionados. $25 \mathrm{~mL}$ de água de osmose reversa à $80{ }^{\circ} \mathrm{C}$ e $20 \mathrm{~mL}$ de ácido sulfúrico $2 \mathrm{~mol} \mathrm{~L}^{-1}$. Essa solução foi titulada com a solução de permanganato de potássio até o aparecimento de uma coloração fraca rósea permanente.

Foram pipetados $5 \mathrm{~mL}$ da amostra de peróxido de hidrogênio, em um balão de $200 \mathrm{~mL}$, completando-se com água de osmose reversa. Em 2 erlenmeyers de $250 \mathrm{~mL}$, foram pipetados $25 \mathrm{~mL}$ da amostra diluída e adicionado ácido sulfúrico 1:1 (v/v). Titulou-se com a solução padrão de permanganato de potássio $\left(\mathrm{KMnO}_{4}\right)$ $0,00225 \mathrm{~mol} \mathrm{~L}^{-1}$ até o aparecimento de uma fraca coloração rosácea permanente (GOBBI e FRIEDRICH, 2011).

\subsection{Comprimento de onda de máxima absorção}

Determinou-se o comprimento de onda $(\lambda)$ de máxima absorção a partir de uma solução de
$10 \mathrm{mg} \mathrm{L}^{-1}$ do corante e foi feita uma varredura em espectrofotômetro Kasuaki IL-592 UV-Vís iniciando em 350 nm e terminando em 750 nm.

A espectrofotometria derivativa consiste na representação das derivadas da absorvância em relação ao comprimento de onda, em função do comprimento de onda. A diferenciação da lei LambertBeer mostra que as derivadas são sempre proporcionais às concentrações do analito, sendo as aplicações analíticas baseadas neste fato (ROCHA e TEIXEIRA, 2004).

\subsection{Curva colorimétrica}

Foi feita uma solução mãe de $10 \mathrm{mg} \mathrm{L}^{-1}$ do corante Rodamina B e a partir da mesma foram feitas diluições e lidas em espectrofotômetro Kasuaki IL-592 UV-Vís para determinar a equação da reta e analisar a degradação da cor que cada tratamento apresentou.

\subsection{Aplicação do Fenton}

Para o tratamento Fenton foi utilizado um béquer de vidro de $1500 \mathrm{~mL}$ contendo $1200 \mathrm{~mL}$ de efluente simulado e barra magnética, sobre agitador magnético. Foi verificado $0 \mathrm{pH}=4,0$, adicionado $0,65 \mathrm{~mL}$ de catalisador sulfato de ferro II heptahidratado $0.1 \mathrm{~mol} \mathrm{~L}^{-1}$ $\left(\mathrm{FeSO}_{4} .7 \mathrm{H}_{2} \mathrm{O}_{(\mathrm{aq})}\right)$ e $15 \mathrm{~mL}$ de peróxido de hidrogênio.

\subsection{Aplicação do foto-Fenton}

Para o tratamento foto-Fenton foi utilizado um béquer de vidro com capacidade de $2000 \mathrm{~mL}$ contendo uma barra magnética, uma lâmpada ultravioleta (UV) dentro de um tubo de quartzo e, $1500 \mathrm{~mL}$ de efluente simulado com $\mathrm{pH}=3.6$ e 4.0 $\mathrm{mL}$ de peróxido de hidrogênio, sobre um agitador magnético.

\subsection{Aplicação da ozonização}

Para o tratamento de ozonização foi conectado um funil sinterizado, que por sua vez conectado a um ozonizador com capacidade de $100 \mathrm{mg} \mathrm{L}^{-1}$ de produção de ozônio, contendo 100 $\mathrm{mL}$ de efluente simulado e $\mathrm{pH}=4.69 \mathrm{com}$ adição de antiespumante devido à formação de espuma.

\section{RESULTADOS E DISCUSSÃO:}

O peróxido de hidrogênio utilizado foi titulado a partir da técnica permanganometria como dito anteriormente e a concentração real obtida foi de $18 \mathrm{~V}$. 
O corante Rodamina B apresentou a leitura sendo $2.001 \mathrm{com}$ maior absorbância em $554 \mathrm{~nm}$ como mostra o gráfico expandido na Figura 1.

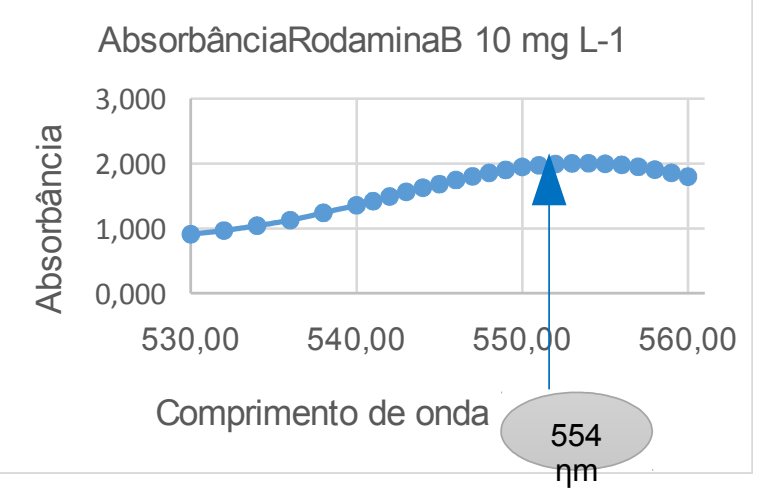

Figura 1. $\lambda$ de maior absorção

A curva colorimétrica foi feita a partir das concentrações e suas respectivas absorbâncias lidas estão representadas na Tabela 1 e na Figura 2.

Tabela 1. Leitura das absorbâncias e suas respectivas concentrações $\left(g L^{-1}\right)$

\begin{tabular}{c|c}
\hline Concentração $\left(\mathbf{g ~ L}^{-1}\right)$ & Absorbância \\
\hline 0.01 & 2.327 \\
0.008 & 1.876 \\
0.0016 & 0.365 \\
0.001 & 0.238 \\
0.0002 & 0.055 \\
0.0001 & 0.002 \\
0.00004 & 0.000 \\
\hline
\end{tabular}

Fonte: Dados primários

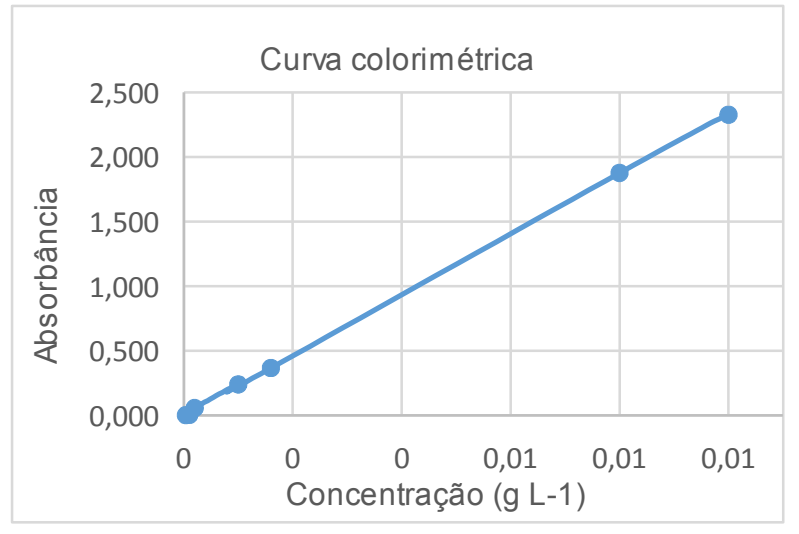

Figura 2. Curva colorimétrica
Para a aplicação do Fenton foi observado a degradação da cor em função do tempo de acordo com a Figura 3, verifica que a descoloração completa ocorre em torno de 25 minutos de tratamento e na Figura 4 pode-se notar a diferença de cor do antes e depois do tratamento.

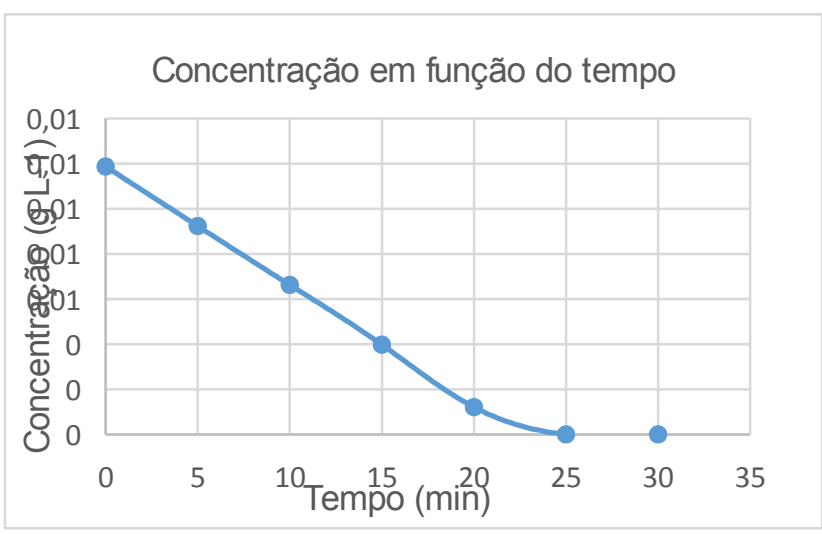

Figura 3. Concentração em função do tempo para o tratamento Fenton

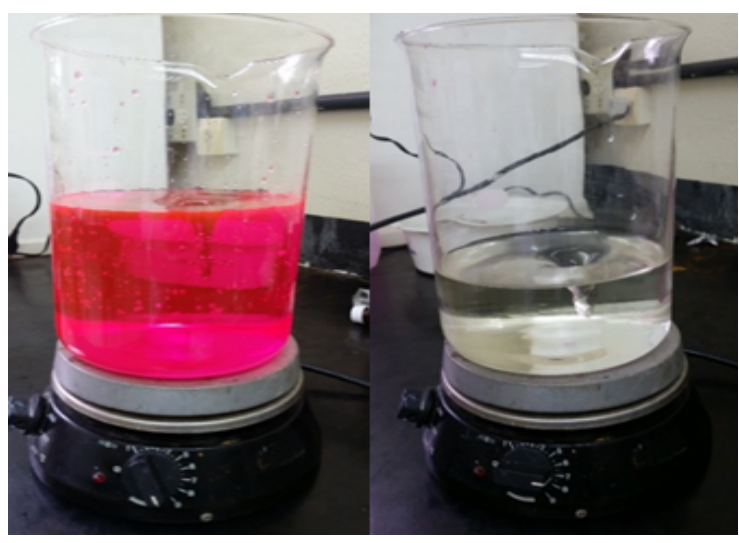

Figura 4. Antes e depois do tratamento Fenton

Para o tratamento foto-Fenton a degradação total da cor pode ser observada no intervalo de 15 a 30 minutos na Figura 5, enquanto na Figura 6 observa-se 0 antes $e$ depois da aplicação do tratamento. 


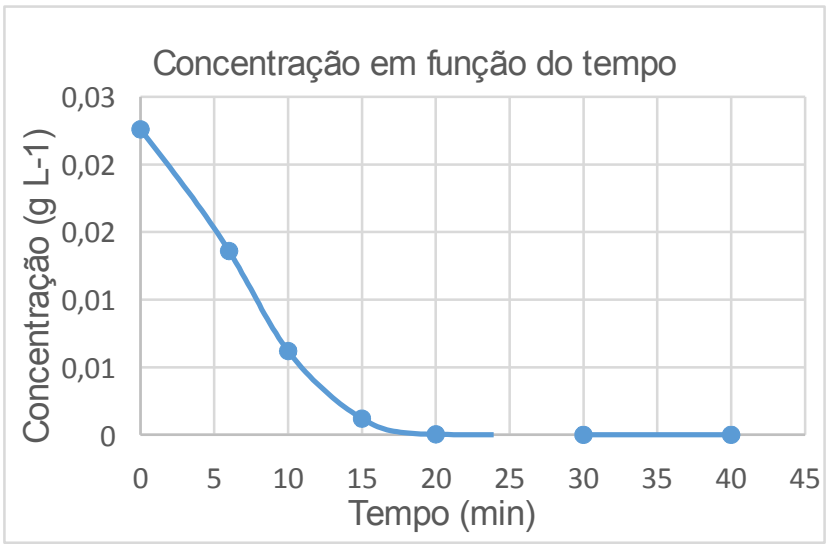

Figura 5. Concentração em função do tempo para o tratamento foto-Fenton

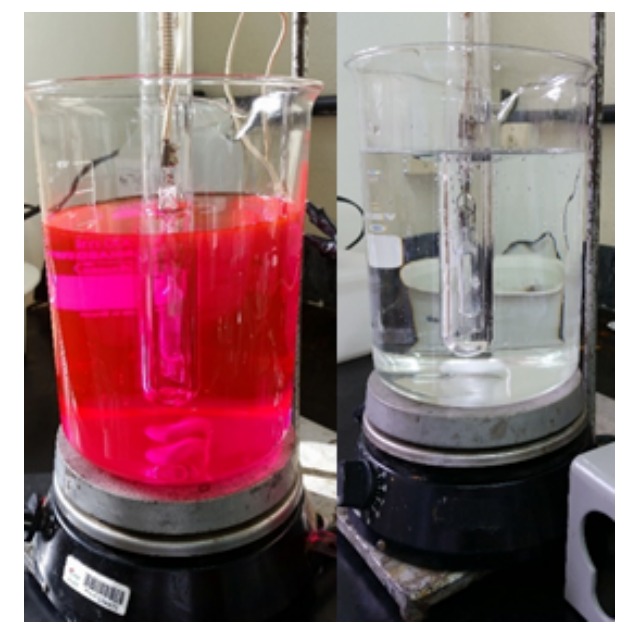

Figura 6. Antes e depois do tratamento fotoFenton

O tratamento da ozonização foi um processo muito rápido, sendo o tempo marcado em segundos e no qual apresentou a degradação completa da cor da Rodamina B como mostra na Figura 7. E na Figura 8 apresenta 0 pré e pós-tratamento. Houve necessidade de adição de 3 gotas de antiespumante na aplicação da ozonização, pois a espuma formada superava a capacidade do funil sinterizado. Este antiespumante acaba gerando um problema, pois aumenta o consumo de oxigênio no efluente.

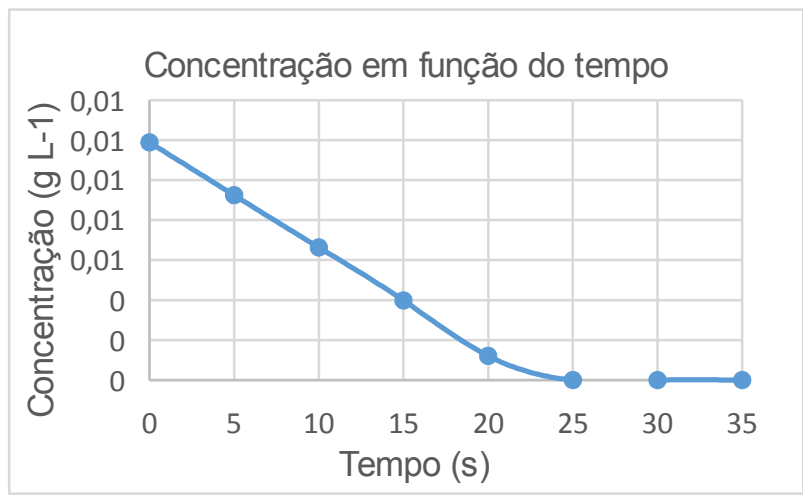

Figura 7. Concentração em função do tempo para o tratamento ozonização

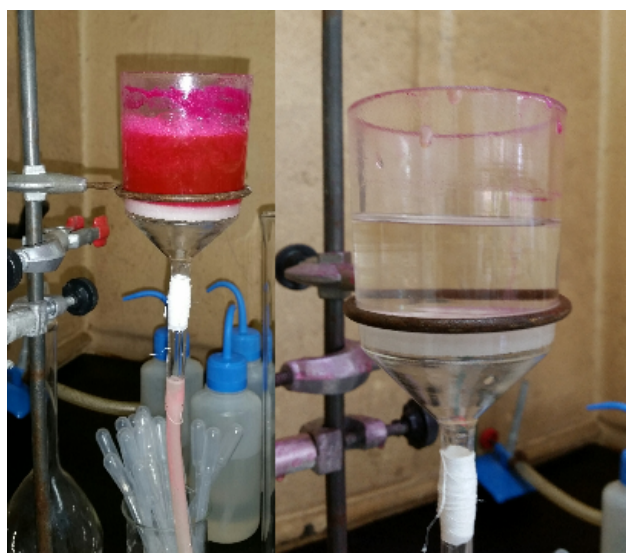

Figura 8. Antes e depois do tratamento ozonização

\section{CONCLUSÕES:}

Os três tratamentos tiveram excelentes resultados na degradação da concentração e consequente degradação da cor do corante Rodamina B. Dentre eles, a ozonização se mostrou mais eficiente que o processo Fenton, porém não foi o mais eficiente de todos, sendo o tratamento foto-Fenton o melhor devido à ser rápido, não necessitando de catalisador como no caso do tratamento Fenton e também por não precisar de antiespumante como no processo de ozonização.

\section{REFERÊNCIAS:}

1. NOGUEIRA, Raquel F. Pupo; TROVÓ, Alam G.; SILVA, Milady Renata A.; VILLA, Ricardo D. Fundamentos e aplicações ambientais dos processos fenton e foto-fenton. Química Nova, Vol. 30 No. 2 p: 400, 2007. 
2. MORAES, H. F., MISTURA, C. M., OLIVEIRA, J. Tratamento de Efluentes Líquidosde tingimento de ágatas, contendo o corante vermelho sangue, através de ProcessosOxidativos Avançados (POAs). (Relatório de Pesquisa). Universidade de Passo Fundo.2011.

3. VIANNA, V; TÔRRES, A; AZEVEDO, E. Degradação de corantes ácidos por processos oxidativos avançados usando um reator com disco rotatório de baixa velocidade. Química Nova, v. 31, n. 6, p. 1353-1358, 2008.

4. APL. Arranjo Produtivo Local de Pedras, Gemas e Joias. Disponível em: $<\& l t$;http://www.portalgemas.com.br/oapl/\&gt>. Acesso em: 05 jun. 2016.

5. IBGM. Características da Cadeia Produtiva Disponível em: <http://www.ibgm.com.br/site/info_cadeia_produtiva.php\&gt>.

Acesso em: 05 jun. 2016.

6. ZANATTA, Alexandre Lazaretti (Org). Plano de Desenvolvimento com Metodologia Participativa APL Pedras, Gemas e Jóias. Passo Fundo: Graffoluz Editora e Industria Gráfica, p. 49, 2014.

7. SERVIÇO NACIONAL DE APRENDIZAGEM INDUSTRIAL (SENAI). Tingimento de Ágatas. Relatório referente ao Projeto GEJORS Desenvolvimento de Novas Tecnologias para o APL de Gemas e Jóias do Rio Grande do Sul, 2008.

8. SALAMONI, Franciele C. Aplicação dos POAs no Tratamento de Efluente Simulado de Indústria de Beneficiamento de Pedras Preciosas. 2008. Projeto de Pesquisa (Graduação em Química) Universidade de Passo Fundo, Passo Fundo, 2008.

9. MARMITT, Sandro; PIROTTA Lilian V.; STÜLP, Simone. Aplicação de Fotólise direta e UV/ $\mathrm{H}_{2} \mathrm{O}$ a efluentes sintético contendo diferentes corantes alimentícios. Química Nova, Vol. 33 No. 2 p: 384-388, 2010.

10. QI, Ping;Lin Z; Li J; Wang C; Meng W; Hong $\mathrm{H}$ e Zhang $\mathrm{X}$. Development of a rapid, simple and sensitive HPLC-FLD method for determination of rhodamine $B$ in chili-containing products. Food Chemistry 164, p. 98-103 China; Elsevier, 2014.

11. PALIWAL, Pradeep; JETTI Srinivasa Rao; BHATEWARA Anjna; KADRE Tanuja e JAIN
Shubba. DABCO - Catalyzed sysnthesis of xanthene derivatives in aqueous media. Laboratory of heterocycles, school of studies in chemistry e biochemistry Vikram University, Ujjan, Madaya-Pradesh. Índia, fev, 2013.

12. ZANONI, Maria Vanilce B.; CARNEIRO, Patrícia A. Odescarte dos corantes têxteis. Ciência Hoje, v. 29, no 174, p. 61-64, abr, 2015.

13. METCALF

EDDY;

(Rev.) TCHOBANOGLOUS, GEORGE; BURTON, FRANKLIN L.; STENSEL, H. DAVID; Wastewater engineering: treatment and reuse. 4th ed. Boston: McGraw-Hill, p. 1819, 2003.

14. LANGLAIS, B., D. A RECKHOW, and D. R. BRINK, 1991 . Ozone in water treatment-application and engineering. American Water Works Association Research Foundation, Denver.

15. HABERL, R., URBAN, W., GEHINGER, P., SZINOVATZ, W. 1993. Oxidation of industrial wastewaters containing non-biodegradable constituents by ozone with and without 69 simultaneous irradiation. In: Proceedings of the eleventh ozone world congress, 29 August - 3 September San Francisco, USA, vol 1, p S10-1 - S-10-12.

16. GOI, Anna. Advanced Oxidation Process for Water Purification and Soil Remediation. 2005. Tese (Doutorado de Filosofia em Engenharia) Tallinn University of Technology, Estônia, 2005.

17. SILVA, Cleber Florêncio da (org.), FRANCO, Paulo, SOUZA, Marcos da Cunha e, ENERAL, Débora Cristina (org.). Responsabilidade civil e penal ambiental, aspectos processuais e licenciamentos ambientais. Curitiba: Intersaberes, p. 38, v. 7, 2014.

18. CAVALCANTI, José Eduardo W. de A. Manual de tratamento de efluentes industriais.2. ed. São Paulo: Engenho, p. 432, 2012. 19. ROCHA, Fábio R. P. e TEIXEIRA, Leonardo S. G.. Estratégias para aumento de sensibilidade em espectrofotometria UV-VIS, 17 jun 2004.

20. GOBBI, Delton Luiz e FRIEDRICH, Maria Tereza. Atividades Experimentais de Química Analítica. Ed Berthier. Passo Fundo, RS. P. 108, 2011. 